\title{
D-ACE surgical sequence for selected bullous retinal detachments
}

\author{
CLARE GILBERT AND DAVID MCLEOD \\ From the Surgical Vitreoretinal Unit, Moorfields Eye Hospital, London EC1
}

SUMMARY Forty-five out of 50 selected retinal detachments were successfully reattached by the D-ACE surgical sequence, that is, initial drainage of subretinal fluid followed by air injection into the vitreous, transcleral cryopexy, and definitive scleral buckling. The D-ACE sequence is recommended for difficult bullous detachments as a simple, safe, and effective alternative to nondrainage techniques.

Experienced retinal surgeons report success rates of over $90 \%$ reattachment (after one or more procedures) in large series of heterogeneous retinal detachments treated by scleral buckling. Detachments resulting from single small breaks in the preequatorial region are especially easy to treat (usually by a non-drainage technique), while the advent of closed microsurgery combined with internal gas tamponade has substantially improved the prognosis for more difficult problems such as posterior breaks in high myopes. There remain, however, several types of retinal detachment which (i) pose special buckling problems, (ii) are not generally considered for closed microsurgery, and (iii) contribute disproportionately to failed surgery and subsequent development of proliferative vitreoretinopathy (PVR). Bullous detachments from multiple large equatorial breaks represent one such group, because the extensive buckling frequently results in fishmouthing of the breaks. In 1980 a surgical sequence comprising initial subretinal fluid drainage (D), followed by intravitreal injection of air (A), transcleral cryopexy (C), and episcleral explant (E) - hence D-ACE sequence'-was introduced in order to overcome the problems which we had previously encountered in managing these difficult detachments. This report describes the details and advantages of the method, and presents the outcome in 50 consecutive procedures. Correspondence to Mr D McLcod, FRCS, Moorficlds Eyc Hospital,
City Road, London ECIV 2PD.

\section{Material and methods}

Between 1980 and 198450 eyes of 50 patients with bullous retinal detachment underwent surgery by the D-ACE sequence in the Surgical Vitreoretinal Unit at Moorfields Eye Hospital. Half the cases were operated on by the consultant-in-charge and the remainder by various members of the resident surgical staff. Eighteen of the 50 eyes had undergone one or more reattachment procedures previously. The majority ( 30 eyes) harboured multiple, usually equatorial, retinal breaks. Of the remainder nine eyes had single tears deemed unsuitable for nondrainage surgery by virtue of their large size and equatorial location, gross elevation, associated static traction, or a combination of these factors; four eyes had large elevated equatorial holes in the outer leaf of a retinoschisis complicated by retinal detachment; two eyes had $90^{\circ}$ dialyses showing fishmouthing after previous buckling; two eyes had no clearly identifiable breaks preoperatively or at surgery; and three eyes had both peripheral and posterior breaks (a macular break in two eyes and a paramacular tear in one eye). Five eyes had signs of early PVR, and two eyes had retinal incarceration from previous surgery; four eyes were aphakic (two with intraocular lenses). Apart from one eye with retinal dialysis and one with retinoschisis the retinal detachments were all associated with posterior vitreous detachment.

Patients were operated upon under general anaesthesia without nitrous oxide; the anaesthetist was encouraged to hyperventilate the patient to promote choroidal vascular constriction. A subconjunctival 
injection of Mydricaine (atropine, procaine, adrenaline, boric acid, sodium metabisulphite) was given to ensure maximal pupillary dilatation throughout the procedure. After reflection of conjunctiva and Tenon's capsule in quadrants to be treated the recti were secured with loops of $2 / 0$ silk.

\section{DRAINAGE OF SUBRETINAL FLUID}

Subretinal fluid was usually drained just above or below one of the horizontal rectus muscles at the equator. After cautery of the lips of a partialthickness meridional sclerotomy (thus creating a scleral cloaca), the incision was deepened to bare choroid which was lightly cauterised and a mattress suture of 5/0 Ethibond placed through the lips of the sclerotomy. The choroid and Bruch's membrane were then perforated with a sharp 24 gauge needle, and 1-3 ml of subretinal fluid was milked from the globe by intermittent scleral depression while the sclerotomy edges were kept everted and extracted from the globe contour (with consequent prevention of retinal incarceration). The sclerotomy was then securely closed.

\section{INTRAVITREAL INJECTION OF AIR}

Before drainage of the subretinal fluid, air or a mixture of air and sulphahexafluoride (SF6) $20-80 \%$ was drawn up via a Millipore filter into a $5 \mathrm{ml}$ syringe; in three eyes the volume to be injected was less than $1 \mathrm{ml}$, so $100 \%$ SF6 was used. A site for injection was almost invariably chosen 3-4 $\mathrm{mm}$ from the limbus and mid-way between the superior rectus and medial rectus insertions, since this was the easiest site to keep 'high' while still affording internal visualisation by indirect ophthalmoscopy. Immediately following subretinal fluid drainage, and with the contour of the superonasal quadrant maintained by inferotemporal scleral indentation, the sclera and pars plana were penetrated by a 27 gauge needle attached to the syringe and observed in mid-vitreous by indirect ophthalmoscopy. The needle was then partially withdrawn to leave a 1-2 mm intravitreal penetration. Once the injection site was confirmed as being the highest point of the globe, gas was smoothly injected into the gel compartment as a single bubble while the scleral indentation was partially released (Fig. 1); multiple intraocular gas bubbles ('fish eggs') were thereby avoided. The needle was then completely withdrawn once it had been ascertained that the eye remained slightly hypotonic so as to preclude any prolapse of vitreous gel through the injection site (which might otherwise result in distortion of tears) and also to leave space for the scleral buckle.

TRANSCLERAL CRYOPEXY

With the exception of the two full-thickness macular breaks, all retinal breaks were completely surrounded by multiple focal cryoapplications, particular care being taken to treat anteriorly up to the ora serrata in the case of large horseshoe breaks. The iceball was allowed to develop so as to freeze both the pigment epithelium and reapposed neural retina, but overtreatment (potentially aggravated by the thermal insulating effect of the adjacent gas bubble) was avoided by careful ophthalmoscopic monitoring and the rapid defrost capability of the MIRA or Wallach cryoprobes. Cryopexy was also applied

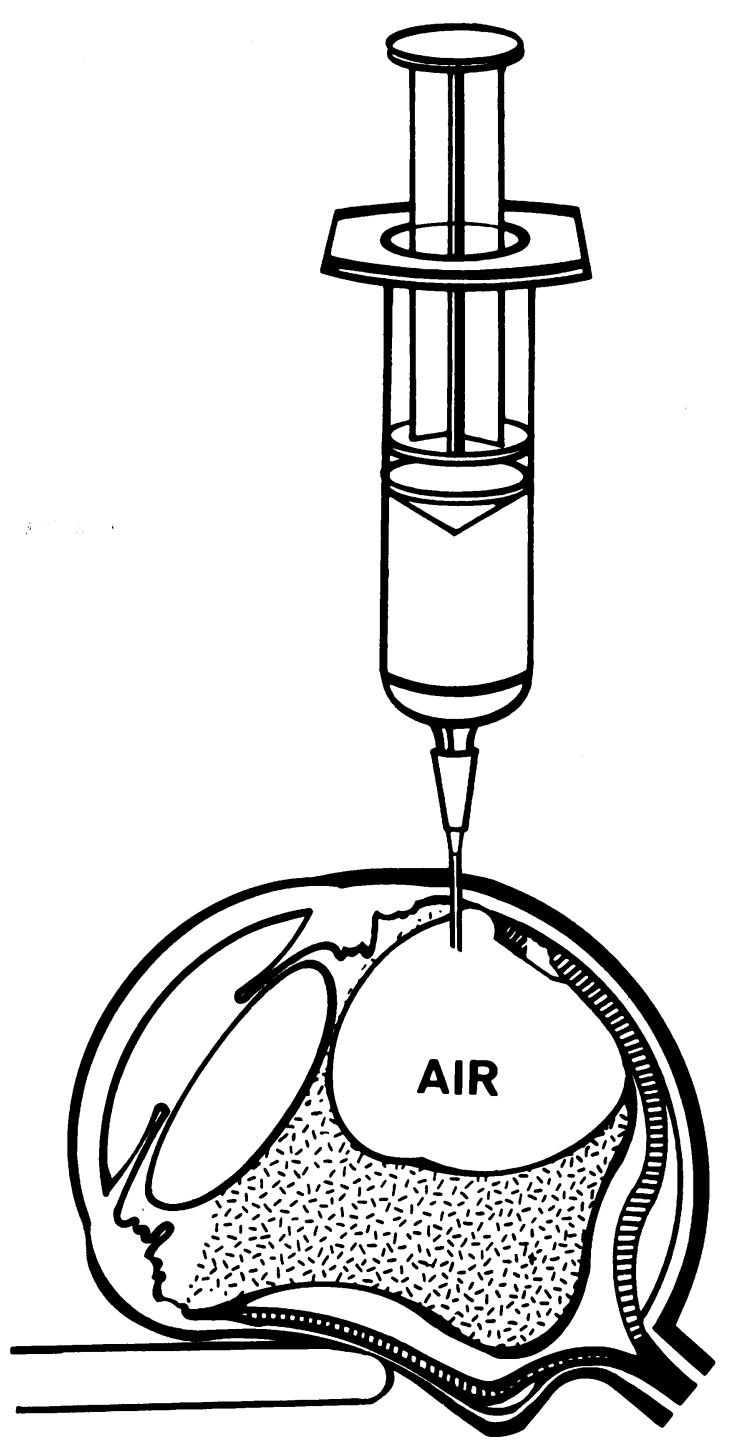

Fig. 1 Needle-tip-high method of intravitreal air injection (after Norton). 
across the posterior margin and lateral edges of other vitreoretinal adhesions-for example, lattice and snail track degeneration-but cryopexy of the subretinal fluid drainage site was avoided wherever possible.

\section{EXPLANT PLACEMENT}

With the exception of the posterior polar breaks in three eyes all retinal breaks were covered externally and indented from their posterior limit up to the ora serrata by means of a 'break-ora occlusive buckle'. This was usually accomplished circumferentially by means of a thin piece of solid silicone material (most frequently a 279 tyre) whose width was chosen (or fashioned) so as to create a gentle $(1 \mathrm{~mm})$ indentation of the sclera by means of hemi-Halsted mattress sutures, each comprising a short $(3 \mathrm{~mm})$ circumferential bite $4 \mathrm{~mm}$ behind the localisation mark of the posterior limit of the most posterior tear, and two radial bites anteriorly through the lateral expansions of the rectus muscle insertions or the intervening scleral ring. The limited height of buckling was controlled by careful matching of suture spread with buckle width and by suture tightening against a well formed globe (by virtue of the gas reconstitution and scleral counter-depression at the start of buckle indentation). The extent of circumferential buckling varied from $60^{\circ}$ to $360^{\circ}$ (mean $170^{\circ}$ ) of the ocular circumference. In three eyes equatorial or postequatorial breaks were mounted on a radially orientated Silastic sponge explant and in four further eyes small postoral breaks were closed by part of a preequatorial encircling 240 band. In 30 eyes an encircling band was used in conjunction with a tyre, and it was otherwise fixed by mattress sutures either across the posterior border of the vitreous base (posterior scleral bite $12 \mathrm{~mm}$ from the limbus) or more posteriorly in the presence of equatorial lattice lesions. The tension in the strap was adjusted via a Watzke sleeve to achieve a low $(1 \mathrm{~mm})$ scleral indentation.

The central retinal artery was then checked, and, if it was closed, an anterior chamber paracentesis was performed or excess gas was tapped off by reelevating the superonasal quadrant and reinserting the 27 gauge needle (closed by means of a finger over the Luer fitting but momentarily reopened once the needle was positioned within the gas bubble). Tenon's capsule was then sutured over the explants to reconstitute the intermuscular septum and the conjunctiva closed. Subconjunctival betamethasone $4 \mathrm{mg}$ and cephaloridine $125 \mathrm{mg}$ were routinely injected.

Postoperatively the patient was positioned so that the arc of contact of the gas bubble included as many of the breaks as possible, and this was maintained for
2-6 days. The minimum follow-up interval after surgery was six months.

\section{Results}

\section{RETINAL REATTACHMENT}

The retina reattached either completely or virtually completely in the immediate postoperative period; in two elderly patients residual inferior subretinal fluid did not become absorbed for two weeks. Transient redetachment occurred on the second postoperative day in two eyes and was attributed to reopening of a break from displacement of the air bubble into the retrohyaloid space.

In 45 eyes retinal reattachment was maintained throughout the follow-up interval and, with a single exception (see below), was associated with visual improvement. The extent of visual recovery reflected the duration of macular detachment, the predetachment acuity, and the age of the patient. The retina became detached again in five eyes, two of which were not reoperated upon. Reoperation (involving vitrectomy, internal gas tamponade, and rebuckling in two eyes, and rebuckling alone in one eye) resulted in successful and permanent reattachment in the other three eyes. Thus retinal reattachment was achieved in $90 \%$ of eyes by means of the D-ACE sequence (subsequently improving to $96 \%$ after reoperation).

\section{COMPLICATIONS}

Significant haemorrhage occurred during surgery in six eyes. In two eyes undergoing reoperation soon after failed cryopexy and buckling, localised subretinal haemorrhage occurred at the time of drainage of subretinal fluid, and a further eye had mild subretinal bleeding from a perforating buckle suture. In one eye a choroidal haemorrhage followed cryotherapy to the site of subretinal fluid drainage adjacent to a large tear; this prevented the completion of cryocoagulation of the tear, which reopened three weeks after initially successful surgery. Finally, two highly myopic eyes developed a choroidal haemorrhage distant from the subretinal fluid drainage site after extensive cryopexy to multiple tears and lattice lesions; the retina reattached in both cases, but in one there was no associated visual improvement:

Serous ciliochoroidal effusions were noted in eight eyes during the first two weeks postoperatively but all settled spontaneously. Transient signs of anterior segment ischaemia, including shallowing of the anterior chamber and folds in Descemet's membrane, were noted in five eyes but required no specific treatment. Each of the following complications occurred once in the series: minimal retinal incarceration requiring no treatment; progressive 
posterior subcapsular lens opacities a year after surgery (and conceivably attributable to gas/lens contact); lens touch from paracentesis causing cataract; and macular pucker. The only eyes to show widespread evidence of PVR were those two eyes in which the retina became redetached and were not reoperated upon.

\section{Discussion}

Successful management of rhegmatogenous retinal detachment demands both identification of all causative retinal breaks and their subsequent closure. Internal closure of breaks by air tamponade per se, as popularised by Rosengren, ${ }^{2}$ may be effective but often only temporarily if there is persistent traction on the breaks (as with horseshoe tears). Chawla et al. reported good results using cryopexy followed by drainage of subretinal fluid, air injection, and equatorial encirclement, ${ }^{34}$ but many of the detachments might have been readily treated by local scleral buckling without drainage of subretinal fluid. The gratifying results of surgery for the problematic detachments in this series are partly attributable to the employment of definitive explants as opposed to equatorial encirclement, which seldom closes the anterior horns of large horseshoe tears effectively. Furthermore, the use of low-profile buckles (which the associated gas tamponade permits) minimises the discomfort, ischaemia, and astigmatisms which so often accompany the high buckles required for nondrainage surgery. Low-profile buckles also reduce the degree of fishmouthing of breaks, and, while fishmouthing is an inevitable complication of extensive buckling for multiple large equatorial breaks, no recourse to special buckling methods ${ }^{6}$ is necessary.

The D-ACE sequence of achieving combined buckling and internal tamponade has a number of theoretical advantages, the validity of which have been born out in practice. The chief complication of subretinal fluid drainage-haemorrhage from the perforation site-is not so much influenced by the site and technique of perforation as by its timing during the course of the surgical procedure. Choroidal vascular congestion induced by cryotherapy renders perforation especially hazardous late into the operative procedure. ${ }^{7}$ Thus drainage of subretinal fluid as the first step in the surgery substantially reduces this risk. Furthermore, provided the introduction of a single large intravitreal gas bubble is successfully accomplished, ${ }^{\mathrm{x}}$ no problem arises in visualising and localising the breaks, which can then be optimally treated by cryotherapy. In order to maximise the benefits of internal gas tamponade while minimising complications, therefore, the decision to forgo non-drainage surgery has to be made preoperatively rather than during the course of the surgical procedure.

The D-ACE surgical sequence has filled a significant gap in our range of surgical options for rhegmatogenous retinal detachment between a nondrainage technique and the employment of vitrectomy - for example, for posterior breaks and for established PVR. When treating difficult detachments which require extensive cryotherapy, we believe that the risk of delayed, incomplete, or failed closure of coagulated breaks after non-drainage surgery poses a greater threat of subsequent PVR than the risk of including the drainage of subretinal fluid in the procedure, as in D-ACE sequence. Not only has the D-ACE sequence improved our success rate for difficult detachments, it has also had a notable effect in reducing subsequent PVR.

We thank Miss Heather Lucas for preparing the manuscript and Miss Karen Johnston for the illustration.

\section{References}

1 McLeod D. D-ACE technique. Presentation to 1st European Silicone Society Meeting, La Baule, June 1982.

2 Rosengren B. Results of treatment of detachments of the retina with diathermy and injection of air into the vitreous. Acta Ophthalmol (Kbh) 1938; 16: 573-9.

3 Chawla HB, Birchall $\mathrm{CH}$. Intravitreal air in retinal detachment surgery. BrJ Ophthalmol 1973; 57: 60-70.

4 Chawla HB, Coleiro JA. Retinal detachment treated with intravitreal air: an evaluation of 241 cases. Br J Ophthalmol 1977; 61: 588-92.

5 Gocl R, Crewdson J, Chignell AH. Astigmatism following retinal detachment surgery. Br J Ophthalmol 1983; 67: 327-9.

6 Pruett RC. The fishmouth phenomenon: II. Wedge scleral buckling. Arch Ophthalmol 1977; 95: 1782-7.

7 Johnston GP, Okun E, Boniuk I, Arribas NP. Drainage of subretinal fluid: why, when, where and how. Mod Probl Ophthalmol 1975; 15: 197-206.

8 Norton EWD. Intraocular gas in the management of selected retinal detachments. Ophthalmology (Rochester) 1973; 77: 85-98. 